\author{
Daniel De Backer \\ Michael R. Pinsky
}

\section{Can one predict fluid responsiveness in spontaneously breathing patients?}

Received: 13 March 2007

Accepted: 27 April 2007

Published online: 17 May 2007

(C) Springer-Verlag 2007

This editorial refers to the articles available at:

http://dx.doi.org/10.1007/s00134-007-0642-y, http://dx.doi.org/10.1007/s00134-007-0644-9, and http://dx.doi.org/10.1007/s00134-007-0646-7

D. De Backer ( $)$

Université Libre de Bruxelles, Department of Intensive Care,

Erasme University Hospital,

Route de Lennik 808, 1070 Brussels, Belgium

e-mail: ddebacke@ulb.ac.be

Tel.: +32-2-5553380

Fax: +32-2-5554698

M. R. Pinsky

University of Pittsburgh, Department of Critical Care Medicine, University of Pittsburgh Medical Center,

Pittsburgh Pa., USA

Prediction of fluid responsiveness has become a topic of intense interest. Although measurements of preload, by whatever technique, are still commonly used to guide fluid therapy $[1,2]$, these fail to estimate the response to fluids in one-half of the patients [3]. Accordingly, many patients may be subjected to the hazards of fluids [4], without benefiting from hemodynamic improvement.

In patients receiving mechanical ventilatory support predictable heart lung interactions can be used to accurately identify fluid responsiveness by noting the arterial pulse pressure or aortic stroke volume variation over three or more breaths [5]. This is because positive-pressure inspiration induces cyclic increases in right atrial pressure causing in turn inverse changes in venous return, right ventricular filling and ejection and ultimately left ventricular preload. In preload-dependent patients these cyclical changes in ventricular filling induce cyclic changes in stroke volume and arterial pulse pressure, provided that both the right and left ventricles are preload responsive. These cyclic changes in pressure and flow are referred to as pulse pressure variation $(\Delta \mathrm{PP})$ and stroke volume variation $(\Delta \mathrm{SV})$, respectively. In spontaneously breathing patients the situation is more complex. Spontaneous inspiration induces negative cyclic changes in intrathoracic pressure causing right atrial pressure to also decrease increasing the pressure gradient for venous blood flow. In calves with total artificial hearts spontaneous ventilation induced opposite changes in left ventricular stroke volume than mechanical ventilation [6]. However, unlike artificial hearts whose right and left side filling is independent of each other, in patients with normal hearts $\Delta$ PP failed to predict preload response during under either pressure support [7, 8] or spontaneously breathing [8]. Various factors may explain this lack of reliability. Both pressure support ventilation and spontaneous breathing are associated with variability in tidal volume, and both $\Delta \mathrm{PP}$ and $\Delta \mathrm{SV}$ are dependent on tidal volume $[9,10]$. Similarly, spontaneous inspiratory efforts may increase intra-abdominal pressure because of active compression of abdominal muscles, exaggerating the preload response. Finally, sudden increases in right ventricular end-diastolic volume decrease left ventricular diastolic compliance by the process of ventricular interdependence, which may decrease left ventricular filling and stroke volume independent of preload-responsiveness, because even failing hearts need some end-diastolic volume to generate stroke volume. Soubrier et al. [11] in their contribution to Intensive Care Medicine now reason that forced expiratory maneuvers (Valsalva maneuver) may identify preload responsiveness in spontaneously breathing patients when normal spontaneous ventilation do not. Using standardized amplitude of respiratory movements they compared the effects of both a Valsalva maneuver and spontaneous 
breathing on $\Delta \mathrm{PP}$ to predict preload responsiveness in 32 critically ill patients. During regular spontaneous breathing activity $\Delta \mathrm{PP}$ was higher in responders to fluid challenge than in nonresponders, but the test performed poorly with a sensitivity of $\Delta \mathrm{PP}$ greater than $12 \%$ of only $63 \%$ although specificity was good $(92 \%)$. The forced maneuver failed to improve the performance of the test, with a decrease in sensitivity to $21 \%$ but a maintained specificity (92\%). Importantly, the cutoff value of $\Delta \mathrm{PP}$ increased to $33 \%$ during the forced expiratory maneuver, indicating that during this large swing in pleural pressure moderate fluctuations in arterial pressure may not indicate fluid responsiveness. This may be due to several factors. First, applying larger tidal volumes and more negative pleural pressure may transiently shift the patient to preload dependency, which disappears when the patient breaths normally. Second, the Valsalva maneuver may also affect right and left ventricular afterload, which may contribute to respiratory variations in stroke volume [12]. Thus, regrettably, $\Delta \mathrm{PP}$ and other derived indices cannot be used in spontaneously breathing patients, as slight and sometimes undetected changes in breathing pattern may affect these variables.

Another way to predict fluid responsiveness is to perform an endogenous fluid challenge using passive leg raising. This approach has been used by cardiologists in the cardiac catheterization laboratory for over 50 years. In critically ill patients under mechanical ventilation, with or without spontaneous respiratory movements, an increase in mean aortic flow of more than $10 \%$ during passive leg raising reliably predicts the response to fluid challenge [7, $13,14]$. Until now this test has been difficult to apply in conscious and spontaneously breathing patients. Passive leg raising requires the use of a fast response measurement of cardiac output as the increase in cardiac output is transient and may not be maintained when legs are raised for more than a few minutes. Hence esophageal Doppler measures of descending aortic blood flow was used in the three reported studies [7, 13, 14]. Unfortunately, using an esophageal flow probe may be quite uncomfortable in conscious patients. To address this issue both Lamia et al. [15] and Maizel et al. [16] in their contributions to Intensive Care Medicine demonstrate that transthoracic echocardiography can be used to measure mean cardiac output during leg raising. They report that an increased change in aortic flow, measured as velocity time interval at the aortic valve (VTIao) by 10-13\%, was associated with a positive response to fluid challenge. In both cases the performance of the tests was very good (receiver operating characteristic curve area of 0.96 [15] and 0.90 [16]) with sensitivity and specificity above $80 \%$. Importantly, one-half of the patients in the study by Lamia et al. [15] and all patients in the study by Maizel et al. [16] were spontaneously breathing without any mechanical support.

These studies provide several lessons. First, changes in mean arterial pressure during passive leg raising failed to predict the response to fluid. Similarly, changes in arterial pressure during fluid challenge were only loosely related to changes in cardiac output, again demonstrating that the only way to assess a positive response to fluid in a spontaneously breathing subject is to measure the changes in cardiac output by whatever the technique. Second, changes in left ventricular area or mitral Doppler, reflecting left ventricular preload, were not useful for predicting the response to fluids, indicating again that preload-responsiveness is not the same as preload.

Several limitations of these last two studies should be acknowledged. First, VTIao rather than stroke volume or cardiac output was measured in order to limit errors in calculation. Both studies considered that aortic diameter did not change during passive leg raising and fluid challenge, and thus VTIao reflected stroke volume. However, if passive leg raising induced increased flow and also increased arterial pressure, aortic diameter may also increase, reducing the accuracy of the VTIao to track flow changes [17]. This limitation may not apply to the aortic outflow tract where VTIao is obtained with echocardiography, as this area is somewhat protected by the aortic annulus. Also, although tachycardia is common in hypovolemia, many studies have reported that there is no major change in heart rate during fluid challenge, even in responders, and therefore stroke volume can be used to assess cardiac output changes, which define fluid responsiveness. Second, some patients experienced a significant decrease in cardiac output both during passive leg raising and fluid challenge. This may reflect a stress-induced change in metabolic requirements or reflect vasoconstriction occurring between baseline and subsequent passive leg raising measurements. This underscores the axiom that hemodynamic evaluation should always be performed carefully in conscious patients and that external factors may interfere with the hemodynamic response to an intervention. Finally, although reliable in experts hands (the inter- and intraobserver variability of VTIao were lower than 5\% in both studies), investigators less experienced with echocardiographic techniques may not reach this level of accuracy. In particular, small changes in the angle of the echo beam may induce errors in measurements that may be misinterpreted (a $15^{\circ}$ angle inducing a 5\% error in measurement). More importantly, echocardiography is not always available at any time of the day and cannot be used for continuous monitoring needed for trend analysis. It is likely that other cardiac output measurement techniques, such as pulse contour determinations, would provide similar results and may supplant echocardiography in bedside monitoring of dynamic changes in cardiac output.

Hence the prediction of fluid responsiveness is now feasible in spontaneously breathing patients, but this requires the performance of a passive leg raising test and the dynamic measure of changing cardiac output using fast response measurements techniques. In the hands of 
the unskilled, much caution needs to be placed on the interpretation of these parameters, but when the data are accurately collected, it represents a new and powerful tool in the critical care diagnostic armamentarium.

\section{References}

1. Rivers E, Nguyen B, Havstadt S, Ressler J, Muzzin A, Knoblich B, Peterson E, Tomlanovich M (2001) Early goal-directed therapy in the treatment of severe sepsis and septic shock. N Engl J Med 345:1368-1377

2. Goepfert MS, Reuter DA, Akyol D, Lamm P, Kilger E, Goetz AE (2007) Goal-directed fluid management reduces vasopressor and catecholamine use in cardiac surgery patients. Intensive Care Med 33:96-103

3. Michard F, Teboul JL (2002) Predicting fluid responsiveness in ICU patients: a critical analysis of the evidence. Chest 121:2000-2008

4. Wiedemann HP, Wheeler AP, Bernard GR, Thompson BT, Hayden D, deBoisblanc B, Connors AF Jr, Hite RD, Harabin AL (2006) Comparison of two fluid-management strategies in acute lung injury. $\mathrm{N}$ Engl $\mathbf{J}$ Med 354:2564-2575

5. Pinsky MR (2004) Using ventilationinduced aortic pressure and flow variation to diagnose preload responsiveness. Intensive Care Med 30:1008-1010

6. Fukamachi K, Irie H, Massiello A, Chen JF, Crouch R, Utoh J, Harasaki H, Golding LA, Kiraly RJ (1992) Effects of mechanical ventilation and spontaneous respiration on hemodynamics in calves with total artificial hearts. ASAIO J 38:M493-M496
7. Monnet X, Rienzo M, Osman D, Anguel N, Richard C, Pinsky MR, Teboul JL (2006) Passive leg raising predicts fluid responsiveness in the critically ill. Crit Care Med 34:1402-1407

8. Heenen S, De Backer D, Vincent JL (2006) How can the response to volume expansion in patients with spontaneous respiratory movements be predicted? Crit Care 10:R102

9. Reuter DA, Bayerlein J, Goepfert MS, Weis FC, Kilger E, Lamm P, Goetz AE (2003) Influence of tidal volume on left ventricular stroke volume variation measured by pulse contour analysis in mechanically ventilated patients. Intensive Care Med 29:476-480

10. De Backer D, Heenen S, Piagnerelli M, Koch M, Vincent JL (2005) Pulse pressure variations to predict fluid responsiveness: influence of tidal volume. Intensive Care Med 31:517-523

11. Soubrier S, Saulnier F, Hubert H, Delour P, Lenci H, Onimus T, Nseir S, Durocher A (2007) Can dynamic indicators help the prediction of fluid responsiveness in spontaneously breathing critically ill patients? Intensive Care Med. DOI 10.1007/s00134-007-0644-9

12. Buda AJ, Pinsky MR, Ingels NB Jr, Daughters GT, Stinson EB, Alderman EL (1979) Effect of intrathoracic pressure on left ventricular performance. N Engl J Med 301:453-459
13. Boulain T, Achard JM, Teboul JL, Richard C, Perrotin D, Ginies G (2002) Changes in BP induced by passive leg raising predict response to fluid loading in critically ill patients. Chest 121:1245-1252

14. Lafanechere A, Pene F, Goulenok C, Delahaye A, Mallet V, Choukroun G, Chiche J, Mira J, Cariou A (2006) Changes in aortic blood flow induced by passive leg raising predict fluid responsiveness in critically ill patients. Crit Care 10:R132

15. Lamia B, Ochagavia A, Monnet $X$, Chemla D, Richard C, Teboul JL (2007) Echocardiographic prediction of volume responsiveness in critically ill patients with spontaneously breathing activity. Intensive Care Med. DOI 10.1007/s00134-007-0646-7

16. Maizel J, Airapetian N, Lorne E, Tribouilloy C, Massy Z, Slama M (2007) Diagnosis of central hypovolemia by using passive leg raising. Intensive Care Med. DOI 10.1007/s00134-007-0642-y

17. Monnet X, Chemla D, Osman D, Anguel N, Richard C, Pinsky MR, Teboul JL (2007) Measuring aortic diameter improves accuracy of esophageal Doppler in assessing fluid responsiveness. Crit Care Med 35:477-482 